\title{
Enlacer l'ajnabi. Une histoire politique de la danse de couple à Téhéran, 1920-1950
}

Embracing the Ajnabi. A political history of couples dancing in Tehran

(1920-1950)

Ida Meftahi

Traducteur : Aurélie Perrier

\section{OpenEdition}

Journals

Édition électronique

URL : https://journals.openedition.org/clio/13722

DOI : $10.4000 /$ clio. 13722

ISSN : 1777-5299

Éditeur

Belin

Édition imprimée

Date de publication : 1 décembre 2017

Pagination : 111-133

ISBN : 978-2-410-00859-3

ISSN : 1252-7017

Référence électronique

Ida Meftahi, «Enlacer l'ajnabi. Une histoire politique de la danse de couple à Téhéran, 1920-1950 », Clio Femmes, Genre, Histoire [En ligne], 46 | 2017, mis en ligne le 01 décembre 2020, consulté le 01 mai 2022. URL : http://journals.openedition.org/clio/13722 ; DOI : https://doi.org/10.4000/clio.13722 


\section{Enlacer l'ajnabi'. Une histoire politique de la danse de couple à Téhéran, 1920-1950}

Ida MEFTAHI*

«Personne ne sait danser la samba, mais ils s'en sortent tous au tango. Ici les femmes chics boivent de la bière et les jeunes de Téhéran se trémoussent!»

Écrite sur un ton humoristique par le jeune auteur Jalal Ni'matullahi, la citation ci-dessus est extraite de l'article "Téhéran danse », publié en janvier 1951 dans l'hebdomadaire Kaviyan. L'article détaille l'engouement croissant des Iraniens pour les danses de couples européennes au milieu du $\mathrm{XX}^{\mathrm{e}}$ siècle. Ces danses, où des corps d'hommes et de femmes s'entrelaçaient, prenaient place dans l'un des nombreux lieux de sociabilité publique de Téhéran ${ }^{2}$. Ceux-ci comprenaient des hôtels et des cafés situés dans le quartier Lalehzar de Téhéran, ainsi que des clubs semi-privés destinés à une clientèle décrite par Nímatullahi comme formant « un monde aristocrate ». Dansant au son de musiques interprétées par des orchestres très populaires, les femmes fréquentant ces clubs portaient des tenues chics avec des

1 “Ajnabi" comprend deux sens : un étranger, et un na-mahram ou homme qui n'a pas de lien de parenté avec une femme et par conséquent avec qui il lui est permis d'avoir une relation sexuelle.

* Je dédie cet article à ma tante Tooran Siahpoosh. Je remercie Elizabeth Claire, Farzaneh Hemmasi, Paromita Kar et les relecteurs externes pour leurs précieux commentaires.

2 Jalal Ni'matullahi, "Tihran miraqsad» [danse de Téhéran], Kaviyan, 22 Day 1329/12 janvier 1951, 6 . 
cheveux relevés, des bijoux brillants et un maquillage exagéré. Reliant l'origine de ces pratiques de danse au dévoilement forcé des femmes en 1936 sous le règne de Reza Shah Pahlavi (ca 1926-1941), Nímatullahi affirmait que l'occupation de l'Iran durant la guerre en 1941 par les forces alliées représentait «une grande révolution» dans la culture nocturne du pays et dans sa culture de la danse :

[...] lorsque les troupes alliées sont entrées en Iran et se sont mises à danser dans les cafés, les femmes iraniennes ont réalisé qu'elles avaient intérêt à mieux danser que leurs homologues américaines et britanniques; elles ont alors organisé des soirées à leur domicile, où elles dansaient toute la nuit avec des gigolos iraniens, au point que leur technique en tango, en swing, en valse et «Waltz-Boston» a attiré l'attention d'étrangers. Cela a entraîné des amitiés et le mariage d'un certain nombre d'entre elles avec des hommes américains qui sont retournés en Amérique après la guerre... Les femmes qui sont restées en Iran ont continué à danser... ${ }^{3}$

Certains lecteurs trouveront peut-être cette description de la vie nocturne de Téhéran surprenante pour un pays musulman au milieu du $\mathrm{XX}^{\mathrm{e}}$ siècle. En examinant des revues de convictions idéologiques différentes, des documents diplomatiques, et des sources d'archives, cet article étudie l'émergence des danses de couple à Téhéran dans la première moitié du $\mathrm{XX}^{\mathrm{e}}$ siècle. Il s'attache également à révéler les craintes nationalistes suscitées autour des questions de genre suite à l'irruption des troupes alliées dans l'espace urbain et social de Téhéran. Enfin, il s'agira de resituer les discours sur les performances de genre et les pratiques de danses de couple «modernes» dans le contexte des transformations politiques de l'époque. Ce faisant, nous analyserons la relation entre le corps social dansant et la notion de modernité (imitée), d'érotisme (shahvat), de nudité et de prostitution, que de nombreux commentateurs contemporains associaient à une forme de complicité avec le colonialisme et l'impérialisme menaçant à la fois l'âme de l'Iran et de l'Islam.

3 Ibid. 


\section{Performer la « modernité » : une notion au cœur de l'Iran du début du $\mathrm{XX}^{\mathrm{e}}$ siècle}

Dès le milieu du $\mathrm{XX}^{\mathrm{e}}$ siècle, les performances sur scène furent reconnues comme un moyen moderne d'éveiller la conscience politique et nationale du public iranien. Le théâtre, tout comme d'autres types de spectacles et évènements, prit une tonalité distinctement politique suivant la Révolution constitutionnelle (1905-1911) qui tenta de limiter le pouvoir de la dynastie Qajar (1785-1926) et d'endiguer l'ingérence politique des empires britanniques et russes dans les affaires intérieures de l'Iran ${ }^{4}$. Ces efforts aboutirent rapidement à la formation d'une communauté d'intellectuels et d'artistes venant de divers horizons ethniques et confessionnels qui s'adonnèrent à toutes sortes d'expérimentations sur scène, bouleversant ainsi le paysage théâtral et transformant un espace qui était auparavant consacré aux distractions en un espace pédagogique dédié à l'édification de la société.

À Téhéran, ces projets se concentraient dans ou aux alentours du quartier Lalehzar. Représentant la quintessence de la modernité urbaine et située à proximité des ambassades et légations principales, la rue Lalehzar et ses environs était le foyer de nombreuses maisons d'édition, théâtres et écoles missionnaires ainsi que d'une gamme de commerces possédés par des Iraniens et des étrangers, et comprenant des boutiques de mode, des magasins d'alcool, des marchands de cigarettes, des hôtels, cafés et restaurants. Une vague d'artistes émigrés venus du Caucase du Sud suite à la Révolution russe de 1917 contribua à enrichir les activités culturelles de Téhéran en apportant leurs compétences propres et leur expérience de la scène ${ }^{5}$. La multiplicité des influences culturelles qui agissaient à la fois sur la performance et la réception du théâtre à Téhéran, est illustrée par une affiche publicitaire (fig. 1) annonçant la tournée d'une troupe venue de Leningrad en perse, russe, français et arménien. La scène artistique étant investie dans l'édification nationale, le contact de la communauté d'artistes avec les idées de modernité et de nation

4 Dans les années 1940, les marxistes et les gens de gauche se tournèrent également vers le théâtre et les performances comme moyen d'élever la conscience politique de leur public. Meftahi 2016 ; voir aussi Guran 1981 : 102.

5 Meftahi 2016. 
engendra la formation de nouveaux genres de danse, telle la danse iranienne nationale (raqs-i-milli), chorégraphie inspirée du ballet, mais mêlant des motifs et thèmes iraniens ${ }^{6}$.

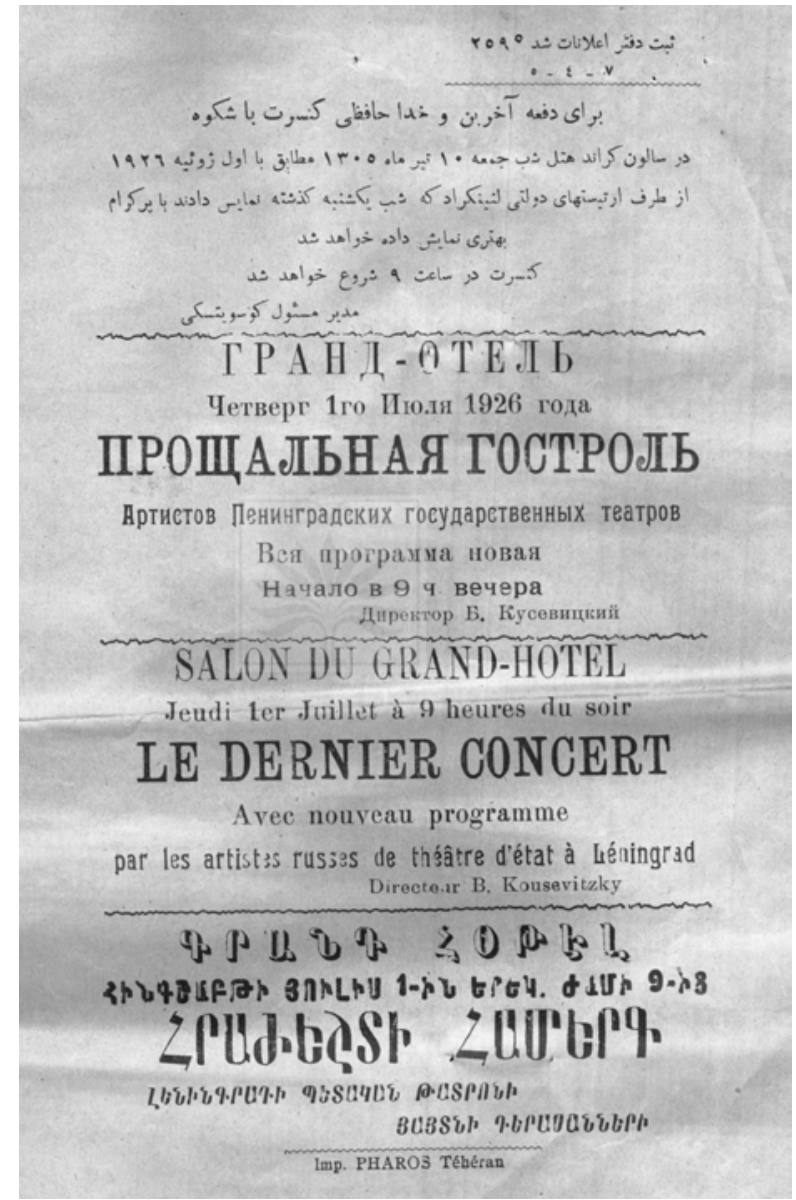

Fig. 1. "Bara-yi daf'ah'i akharin va khudahafizi-i bashukuh" [For the last time and farewell a glorious concert], publicité pour concert,

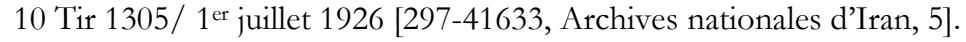

6 Meftahi 2016. 
Avides de modernité, les membres appartenant à cette sphère culturelle cosmopolite étaient adeptes de formes de sociabilité et de pratiques corporelles non-iraniennes. Ils partageaient également avec les communautés étrangères les lieux de spectacle et de loisirs situées autour du quartier Lalehzar. Ces pratiques étaient souvent en tension avec un ethos nationaliste et avec l'ordre social local, expliquant qu'en 1914 le ministre de l'Intérieur décida d'imposer une autorisation obligatoire pour tout événement public incluant des concerts, des danses de couple et des soirées tenues à la fois par des Iraniens et des étrangers 7 .

Malgré cette mesure, au milieu des années 1920 lorsque Reza Shah abolit la dynastie Qajar et se couronna premier monarque Pahlavi, les danses de couple mixtes étaient devenues un élément habituel des soirées publiques, bals masqués, spectacles de variété et performances diverses tenues dans le quartier Lalehzar ${ }^{8}$. Dans un contexte où les femmes musulmanes apparaissaient généralement en voile intégral dans l'espace public, les danses entre hommes et femmes représentaient une pratique scandaleusement peu orthodoxe. Même sur la scène de spectacle présumée avant-gardiste, les rôles féminins étaient joués par des femmes non-musulmanes ou par des hommes habillés en femme. La danse de couple attira donc peu à peu des critiques, parfois sous la forme de poésies exhortatoires, comme ce poème de 1925 intitulé « La femme et la chasteté », dans lequel le poète demande à sa bien-aimée de ne pas danser avec tous les hommes ni dans tous les lieux, car loin d'être le signe de son sens artistique, cela entacherait son caractère?

7 Du commissaire de police de Téhéran au ministre de l'Intérieur iranien, 11 octobre 1914, 293-1303, Archives nationales d'Iran, 4.

8 Voir par exemple, «Ti'atr-i namus» [Le jeu d'honneur], Rabnama, 183, 28 Zihajjah 1338/12 septembre 1920, p. 4, réimprimé dans Kouhestani-Nejad 2002 : 236-237 ; «Kunsir-i Urupa'i », Ittila'at, 3 Bahman 1307/23 janvier 1929, p. 2 ; «Kafah'i luks » [Cafés de luxe], Shafaq-i Surkh, 289, 14 Day 1303/4 janvier 1925, p. 4, réimprimé dans Mas'ud Kouhestani-Nejad 2005 : 312 ; «Barguzari-i kusirt dar hutil du france shab-i dushanbah 6 rajab sa'at 8 » [Concert à l'Hôtel de France lundi 15 mars à 20h00], Iran, 1061, 14 Isfand 1300/5 mars 1922, p. 4, réimprimé dans Kouhestani-Nejad 2005 : 139.

9 Dr. Afshar, "Zan va 'iffat » [Les femmes et la chasteté], Iranschabr, 1925, 3/1-2, in Kazmizadeh Iranschahr 1984 : 91-92. 
Invitant ses lecteurs à lire ce type de poésies afin de combattre l'influence négative de l'Europe sur les nouvelles habitudes sociales, l'écrivain Ahmad Kasravi affirmait qu'il «n'y a rien de plus honteux que la danse d'un homme de cinquante ans ${ }^{10}$. Kasravi ajoutait que les hommes iraniens ne pratiquaient pas ou peu la danse en public (y compris les formes de danses locales), sauf sur scène ${ }^{11}$. Par ailleurs, un bref rapport publié dans le quotidien Ittila'at traitant de l'interdiction des danses Charleston et Black Bottom en Italie pour des raisons d'ordre moral et familial, faisait également allusion à la prise de conscience progressive de l'impact «immoral» de ces pratiques sur la communauté « modernisée » de Téhéran ${ }^{12}$.

Cependant, comme l'indique une affiche en français datant de 1926 (fig. 2), l'enthousiasme pour les danses de couple resta suffisamment répandu pour justifier la création du Salon de Danse Olympia, un espace organisant un bal masqué sur de la musique jazz (" et sur du saxophone»), et accordant gratuitement l'entrée aux femmes. Bien qu'aucune mention de l'origine française de l'événement ne figurait sur l'affiche, celui-ci évoquait l'influence culturelle de la France en tant que source d'inspiration et destination historique pour les intellectuels iraniens, ainsi qu'un certain nombre d'établissements du quartier Lalehzar - y compris les deux écoles importantes de Saint Louis et Jeanne D'Arc, le Paris Hotel et le grand magasin Le Bon Marché qui disséminait les modes françaises.

10 Ahmad Kasravi, «Sharq va gharb» [East and West], Armaghan, 144, Isfand 1310/mars 1931, p. 31-35.

11 La pratique de la danse par les hommes était courante parmi les modes de vie ruraux et ethniques de l'Iran.

12 «Mamnu'iyat-i raqs dar italiya » [Interdiction de danse en Italie], Ittila'at, 24 Day 1306/15 janvier 1928, p. 3. 


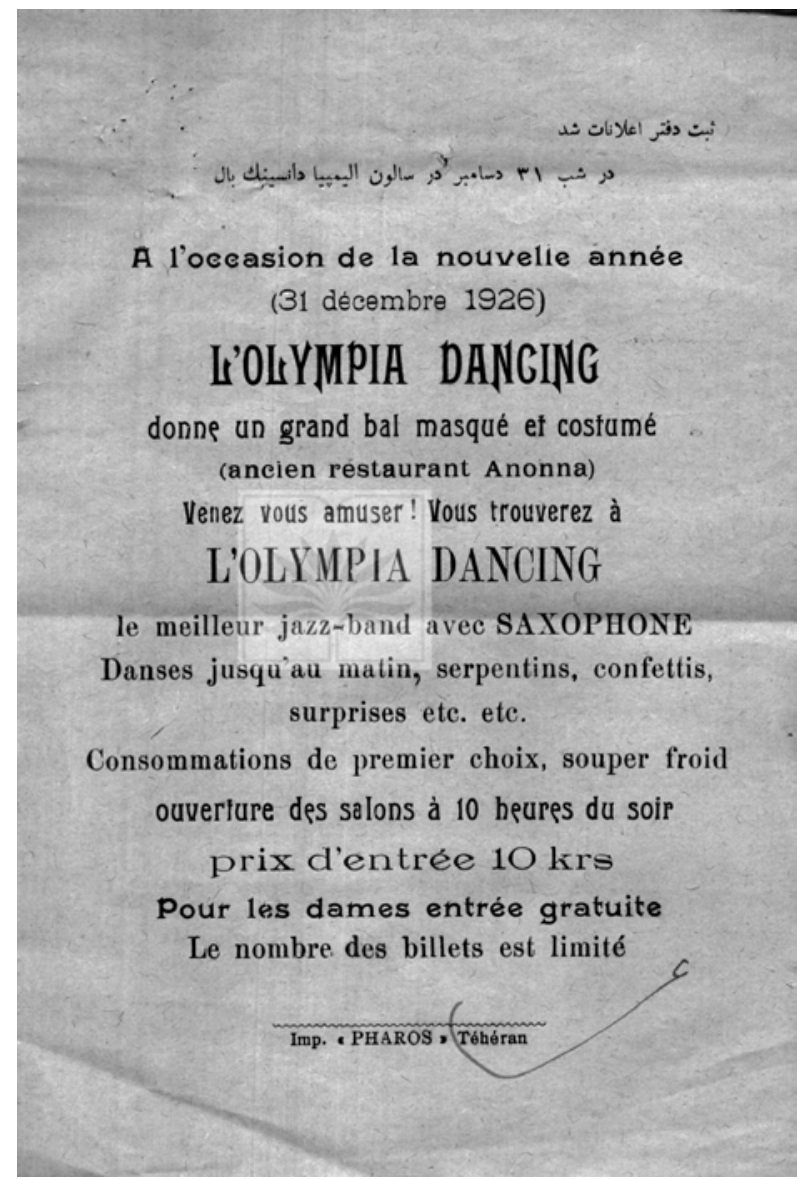

Fig. 2. Poster du Salon de Danse Olympia, 31 décembre 1926 [297-41633, Archives nationales d'Iran, 4]. La translitération perse de "danse-ing" était utilisée sur le poster. 
L'adoption du terme français danse pour désigner les danses de couple soulignait par ailleurs l'engouement pour les tendances et pratiques de loisirs françaises parmi certains cercles iraniens ${ }^{13}$. La scène artistique étant investie dans l'édification nationale, le contact de la communauté d'artistes avec les idées de modernité et de nation engendra la formation de nouveaux genres de danse, telle la danse iranienne nationale (raqs-i-milli), chorégraphie inspirée du ballet, mais mêlant des motifs et thèmes iraniens ${ }^{14}$. La coutume consistant à accorder plus facilement l'entrée aux femmes continua au moins jusqu'en 1932, lorsqu'une publicité pour un «bal glorieux» annonçait des billets moins chers pour les femmes ${ }^{15}$. Parallèlement, l'intérêt journalistique pour les danses de société augmentait, comme en témoigne la publication d'articles et de caricatures reflétant un large éventail d'attitudes en Iran face à ces pratiques de danses ${ }^{16}$.

Le dévoilement forcé des femmes en 1936 faisait partie intégrante du premier Programme d'émancipation des femmes mené par le régime Pahlavi. Ce programme visait à intégrer les femmes dans la société et promouvait en tant que telle la visibilité des femmes au travail, sur la scène artistique et dans les lieux de loisirs. Dans l'ensemble, la presse nationaliste jugeait que la danse représentait une forme d'exercice physique convenable pour les femmes de la nation tant que celle-ci était pratiquée à la maison. Des publicités pour des tutorats en danse commencèrent donc à émerger dans la presse, comme cette publicité pour les cours en studio de Mademoiselle Ujik Baghdasarian parue dans

13 Pour une histoire culturelle de la réception des danses «modernes » du tango et du foxtrot dans le Paris de l'entre-deux-guerres, voir Jacotot 2013 ; et pour la valse «moderne » dans le Paris de la fin du XVIII siècle, voir Claire 2013. Puisque la notion de danse de couple était étrangère au discours public iranien, les termes étrangers de danse et danse-ing, plutôt que les termes courants en Persan de «raqs » (lui-même d'origine arabe), était fréquemment employés, souvent en liaison avec un adjectif tel que « européen », « moderne », ou « social ».

14 Meftahi 2016.

15 «Bal-i ba-shukuh » [Une soirée glorieuse], Ittila'at, 1622, 16 Khurdad 1311/6 juin 1932, p. 1.

16 «Raqs » [Danse], Mihrigan, 79, p. 16 ; «Amukhtan-i raqs (Lambet vak) ziyad giran tamam shudah» [Apprendre la danse (Lambeth Walk) revient très cher], Mibrigan, 84, p. 4. 
le quotidien Ittila 'at et dans Mibrigan, mensuel à l'intention des femmes : l'annonce indiquait la possibilité de prendre des leçons privées (facturées au tarif-horaire ou sur contrat) en plastique ${ }^{17}$ et danses russes, ainsi qu'en «danses modernes» (raqs-ha-yi jadid) enseignées selon la "méthode parisienne » et incluant le foxtrot, le tango et la valse ${ }^{18}$ (fig. 3). En 1939, la liste des cours offerts par Ujik comprenait également le Lambeth Walk, le conga et la rumba.

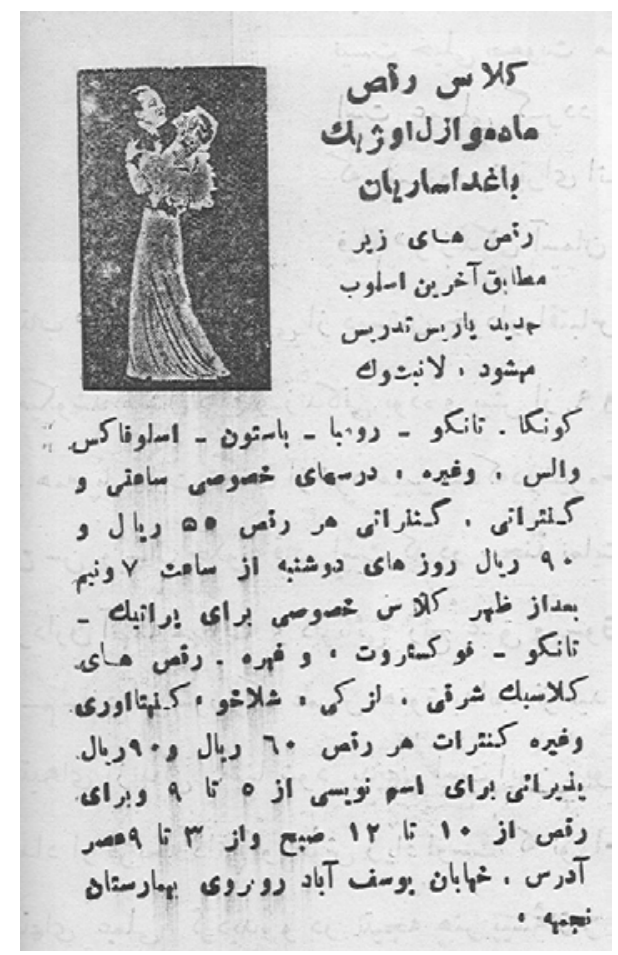

Fig. 3. « Kilas-i raqs-i Mademoiselle Ujhik Baqdasariyan » [Classes de danse de Mademoiselle Ujhik], Mibrigan, Isfand 1318/mars 1940, p. 13.

17 Émile Jaques Dalcroze, Méthode Jaques-Dalcroze. Exercices de plastique animée, vol. I, Lausanne, Jobin \& Cie, 1916 ; voir également, Émile Jaques-Dalcroze, Méthode Jaques-Dalcroze. Marches rythmiques, vol. I, Lausanne, Sandoz-Jobin \& Cie, 1906.

18 «Kilas-i raqs tavassut-i madmazil ujhik» [Le cours de danse de Mademoiselle Ujik]", Ittila'at, 15 Azar 1316/6 décembre 1937, p. 7 ; "Kilas-i raqs-i Mademoiselle Ujhik Baqdasariyan », Mibrigan, Isfand 1318/mars 1940, p. 13. 
La popularité des danses de couple à la fin des années 1930 a dû dépasser rapidement la capacité des cours d'Ujik: en 1940 le premier manuel pour danses de couple, intitulé Manuel des nowvelles danses fut traduit du français par Iraj Akhgar (fig. 4).

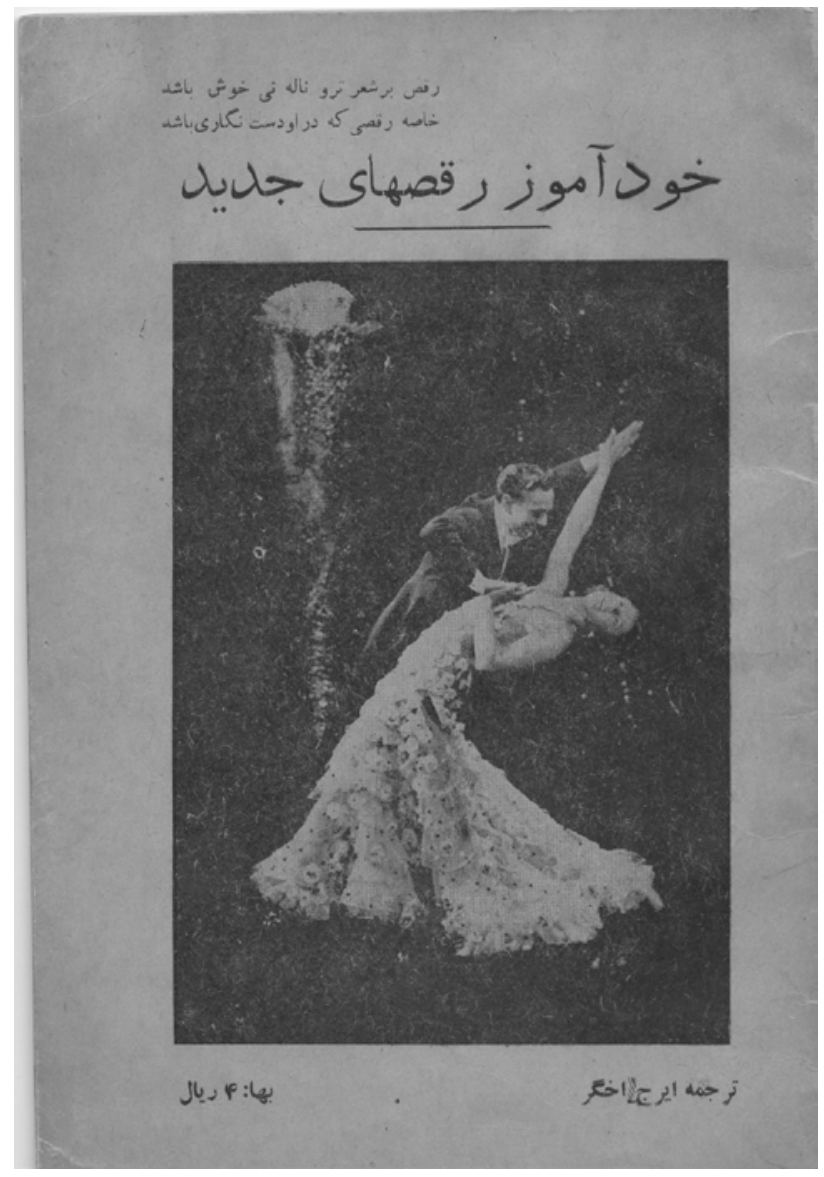

Fig. 4. Couverture de Iraj Akhgar (trans.),

Khud-Amuz-i Raqs-ba-yi Jadid [Manuel des danses nouvelles/modernes], Téhéran, Chapkhanah'i Bahar, 1319/1940. 
Rédigé par Carl Werner, présenté comme un professeur de danse de renommée internationale, ce manuel de quarante-quatre pages fournissait des instructions pour dix danses, y compris pour le onestep, le quick-step, la valse anglaise et le pasodoble. En plus du titre du livre et d'une image illustrant un couple en train de danser, un verset du poète iranien du XIII siècle, Hafiz, figurait sur la couverture : « la danse est une joie lorsque la flûte chante et qu'une fraiche poésie fuse, particulièrement une danse où l'on tient la main de la personne qui nous est chère » (fig. 4). Les réactions hostiles à ces nouvelles pratiques urbaines ainsi qu'à la politique d'émancipation des femmes menée par le premier monarque Pahlavi n'émergèrent pas toutefois avant l'invasion anglo-soviétique de l'Iran en août 1941, lorsque Reza Shah fut écarté du pouvoir puis remplacé par son jeune fils Mohammad Reza (ca 1941-1979), connu en occident comme le Shah.

L'occupation alliée incita la nouvelle presse religieuse politisée à défier la consigne de dévoilement des femmes de 1936. Selon les journalistes appartenant à cette mouvance, la mesure avait conduit à «l'intrusion» de la sexualité féminine dans l'arène publique, provoquant ainsi un érotisme (shahvat) qui était la cause présumée de toutes les obscénités dans la société. Les participants à ces «activités obscènes» étaient décrits comme des hommes et des femmes modernisés (mutajaddid), qu'un ensemble de signifiants visuels et d'actions corporelles genrées venaient distinguer du reste de la société musulmane. Ainsi, une femme mutajaddid était non-voilée et facilement identifiable grâce à sa tenue transparente courte et colorée et le port de maquillage et de vernis à ongle ${ }^{19}$. Décrite par ces auteurs comme «nue» ou «semi-nue», elle aimait flirter, était coquette, et contrairement aux femmes musulmanes «convenables", manquait d'incarner les valeurs de hijab (voilement), iffat (chasteté), 'ismat (pureté) et haya (modestie) ${ }^{20}$. Cette représentation caricaturale de la

19 Hidayatullah Hatami, «Khahran-i musalman » [Sœurs respectables], Nur-i Danish, 3 Day 1326/25 décembre 1947, p. 366 ; «Daf'-i javanan-i vilgard kar-i khubi ast» [Rejeter des jeunes vagabonds, c'est une bonne action], Nida-yi Haq, 20 Day 1334/11 janvier 1956, p. 1.

20 H. Tavassuli, «Shuma kah abiru nadarid » [Oh Vous, gens déshonorants !], Nidayi Haq, 15 Shahrivar 1334/7 septembre 1955, p. 1, p. 4 [citation en 4]. Outre sa fonction de couvrir, le hijab est le symbole d'une qualité intérieure. 
femme moderne qui demandait des droits égaux à ceux de l'homme, pouvait également être reconnue par le fait qu'elle travaillait en dehors de la maison dans un bureau mixte, n'obéissait pas à son mari, avortait quand elle le souhaitait, déambulait dans les espaces publics tels que le cinéma et le théâtre, et faisait de la bicyclette en public ${ }^{21}$.

Les hommes n'étaient pas épargnés par la critique. À la différence des hommes " convenables », les mutajaddid de sexe masculin juraient, consommaient de l'alcool, jouaient au jeu, riaient fort et étaient sexuellement agressifs envers les femmes ${ }^{22}$. De plus, un homme mutajaddid n'hésitait pas à afficher sa femme en public et l'encourageait à se dévoiler; il était donc décrit comme bi-qayrat (dépouillé de zèle) et incapable de protéger son honneur ${ }^{23}$. Ces propos trouvaient leur justification dans une pensée traditionnelle qui considérait la femme comme le namus (honneur) de l'homme musulman, nécessitant alors sa protection ${ }^{24}$. Les hommes se devaient donc de protéger les femmes tout comme ils se devaient de protéger leur pays ${ }^{25}$. Les hommes politiques mutajaddid étaient vus comme les principaux manipulateurs derrière la campagne de dévoilement des femmes. Certains écrivains conservateurs maintenaient même qu'«au nom de la liberté, les hommes ont dupé les femmes et en ont fait leurs jouets $»^{26}$. Ce traitement binaire des identités dans la société

21 «Ma na ifrati hastim na tafriti » [Nous n'allons pas aux extrêmes], Nida-yi Haq, 28 Mihr 1333/20 octobre 1954, p. 1, p. 2 [citation en 2].

22 H. Tavassuli, «Shuma kah», op. cit., p. 4 ; Murtiza Hashimi Hissam-Sari, "Tamaddun va tajaddud, tadayyun va irtija » [Civilisation et modernité, religiosité et sous-développement culturel], Nida-yi Haq, 6 Isfand 1331/25 février 1953, p. 1, p. 2 .

23 Ahmad Shari'ati, "Qurumsaq-ha ra bishinasid» [Faites la connaissance des proxénètes], Dunya-yi Islam, 2 Aban 1326/20 octobre 1947, p. 3.

24 S.J. Tabrizi, «Dar piramun-i hijab » [À propos du hijab], Nur-i Danish, 27 Isfand 1948/18 mars 1948, p. 718-719; «Dar atraf-i hijab va qur'an » [Sur le hijab et le Coran], A'in-i Islam, 27 Murdad 1323/18 août 1944, p. 6.

25 Muhamad-Ali Taqavi, "Millat-i dindar alaqahmand bah hifz-i namus-i khud mibashad » [La nation dévote et soucieuse de la protection de son namus], Dunyayi Islam, 16 Aban 1326/8 novembre 1947, p. 1, p. 4.

26 Yadullah Niyazmand-Shirazi, «Zan dar ijtima'i diruz va imruz » [Les femmes dans la société d'hier et d'aujourd'hui], Nur-i Danish, 9 Mihr 1326/2 octobre 1947, p. 52-53 [citation en 52]. 
iranienne, ainsi que l'attribution du qualificatif d'érotique ou d'obscène à toute une panoplie de pratiques et d'espaces vus comme chargés d'« érotisme» (shahvat), donna lieu à un véritable « discours anti-obscénité » qui était également anticolonial ${ }^{27}$. Ce discours dépeignait les intentions colonialistes et l'imposition de pratiques sociales coloniales en Iran comme la source principale de la corruption morale frappant les Iraniens musulmans. Cette corruption se manifestait précisément dans l'érotisation néfaste des relations hommes-femmes, incarnées par les danses de couple « modernes ».

\section{Danser dans les bras de l'occupant}

Durant la Seconde Guerre mondiale, l'invasion de l'Iran par les Alliés et leur présence sur le sol iranien malgré la déclaration de neutralité de l'Iran, intensifia le sentiment anticolonialiste. Celui-ci était nourri par une opposition à des pratiques genrées attribuées à la «tajaddud» (modernité d'imitation). Durant la guerre, l'honneur iranien (namus) était non seulement métaphoriquement menacé par l'occupation de la mèrepatrie, mais physiquement et moralement affaibli par la promiscuité physique des femmes iraniennes et des occupants masculins. Cette situation était particulièrement bien illustrée par la polémique sur les lieux de loisirs de Téhéran et par les réactions socio-politiques concernant le quartier Lalehzar et ses activités. Téhéran, à la différence de la partie nord de l'Iran sous influence soviétique et de la partie sud du pays sous influence britannique, était située dans une zone neutre. La rue Lalehzar et ses environs devinrent une zone d'affrontements culturels où les pouvoirs alliés suivaient chacun son propre agenda de diplomatie publique. Ces activités allaient de la projection de films américains, aux performances théâtrales subventionnées par les soviétiques, en passant par les soirées shakespeariennes britanniques et les cours d'anglais visant à remplacer le français par l'anglais dans l'Iran

27 Le terme «shahvat» est souvent traduit par «luxure» ou «passion » (en anglais). Je préfère le vocabulaire relevant de «l'éros » qui illustre mieux le sens de shabvat dans le présent contexte. Par ailleurs, le terme «shabvat» correspond historiquement au terme « érotisme ». Meftahi 2016 : 133-143. 
d'après-guerre ${ }^{28}$. Parallèlement, les soldats alliés devinrent une communauté visible dans le quartier Lalehzar: résidant dans les hôtels, ils flânaient à travers les rues, fréquentaient les bars, restaurants et cinémas du voisinage et dansaient dans les cafés au milieu des habitants "modernisés" (mitajaddid) de Téhéran.

La présence des troupes britanniques et américaines sur ces sites de sociabilité "érotiques" ainsi que les interactions entre soldats et femmes iraniennes, formaient pour beaucoup d'Iraniens une situation humiliante. L'image de femmes iraniennes enlacées dans les bras d'étrangers, quelques années à peine après le dévoilement forcé des femmes sous Reza Shah Pahlavi, sonnait comme une provocation et une menace pour l'«honneur» iranien (namus). En outre, la frénésie pour les danses de société à Téhéran était interprétée comme le signe tangible de l'indifférence de la population modernisée à la pauvreté et la famine touchant le reste du pays en raison de la guerre. Le magazine satirique Khurshid-i-Iran publia une caricature (fig. 5 - cahier couleur) décrivant cette situation.

Sur un ton humoristique, un éditorial du même magazine décrivait la danse comme "un fléau» et "une maladie » qui avait semé la pagaille dans le pays, tandis qu'un autre article ridiculisait l'idée que le progrès et la modernité pouvaient s'incarner dans la danse de couple dans le noir ${ }^{29}$.

28 Les États-Unis entrèrent dans la guerre en décembre 1941 et déployèrent des troupes militaires bien qu'ils n'aient pas signé de traité. Les pouvoirs alliés suivaient chacun leur propre agenda de diplomatie publique et de propagande. TNA: BW 49/3/PER/2/1/1, de N.N. Tett, Institut Anglo-Perse de Téhéran au Consul britannique, Londres, 15 novembre 1944: rapport d'octobre de la Société dramatique anglo-iranienne. TNA: BW 49/3, de J.S. Bigley, Consul britannique Téhéran, à Martin Blake, Consul britannique Londres, 1 ${ }^{\text {er }}$ juin 1944 : extrait du Rapport annuel 1943/1944. TNA: BW 49/3, de Hilary Wontrer, Société dramatique anglo-iranienne, au Consul britannique, Londres, 20 avril 1944 : Le théâtre en Iran. Voir aussi Naficy 2011 : 1-48.

29 «Mamlikat-i majanin, maraz-i raqs-i vahdat ya da'ul majanin » [Le pays des fous, la maladie des danses de société, ou bedlam], Khurshid-i Iran, 29 Mihr 1321/21 octobre 1942, p. 6 ; "Ash-i shu'lah qalam-kar» [Mish mash], Khurshid-i Iran, 4 Bahman 1321/24 janvier 1923, p. 2, p. 4. Les réformistes évangéliques et les royalistes britanniques menèrent une campagne similaire contre « les maladies sociales » des danses de couples «modernes» dans la presse satirique durant les 
De plus, le comportement, les actions et les interactions sociales des troupes britanniques et américaines à Téhéran créaient un ressentiment généralisé envers les occupants. Comme l'indiquent plusieurs documents tirés des archives nationales des États-Unis et des archives britanniques et iraniennes, en 1942 il y avait déjà eu plusieurs cas de mauvaise conduite de la part du personnel américain et britannique, engendrant des heurts avec la population locale ${ }^{30}$. En réponse aux pressions sociales, l'armée britannique adressa un nouveau règlement en juin 1942 à l'attention des militaires en poste à Téhéran, comportant des instructions spéciales sur la façon de saluer et de s'habiller ainsi que sur la conduite sexuelle et les attitudes à adopter avec les Iraniens. Le règlement déclarait l'ivresse comme un délit de cour martiale lorsqu'il était commis au Moyen-Orient ${ }^{31}$. Les troupes alliées étaient également mises en garde contre tout acte de socialisation avec les femmes locales et contre la dissimulation de maladies vénériennes ${ }^{32}$. Enfin, certains lieux étaient considérés hors-limite pour les troupes, comme le quartier de prostitution ou le camp de réfugiés polonais (sauf en poste ou si l'on s'y rendait sur invitation), alors que plusieurs lieux du quartier Lalehzar étaient spécifiquement désignés comme acceptables - laissant deviner que des arrangements eurent lieu entre ces commerces et la police urbaine de Téhéran, notamment concernant l'heure de fermeture et la vente d'alcool. Suite à la diffusion du règlement, un Comité social fut

premières années du XIXe siècle alors que la valse allemande s'emparait des bals de société à Londres, voir Claire 2017.

30 Légation américaine de Téhéran, au Secrétaire d'État, Washington; Boite 53, vol. 820.02, 1942 : 820.2-851, 3 ; courriers du Service extérieur du Département d'État (Foreign Service Post), Ambassade de Téhéran, dossier groupe 84 (ci-après abrégé par RG pour Record Group) ; Archives nationales de College Park, College Park, MD. Louis G. Dreyfus Jr., au Secrétaire d'État, Washington, 13 juillet 1943 ; 841.5; courriers du Service extérieur du Département d'État, ambassade de Téhéran, RG 84 ; Archives nationales de College Park, College Park, MD.

31 Règlement pour le personnel militaire de Téhéran, 6 juin 1942; Boite 53, vol. 822, 1942 : 820.2-851 ; Service extérieur du Département d’État, ambassade de Téhéran, RG 84 ; archives nationales de College Park, College Park, MD.

32 Ceux qui étaient diagnostiqués comme étant physiquement compromis par une maladie vénérienne ou par l'alcoolisme perdaient leur droit à la paye de bonne aptitude. 
formé pour la sous-zone de Téhéran le 10 décembre 1942 afin de "préserver le moral de l'armée et répondre aux besoins de divertissement des troupes». Suivant le raisonnement que «si l'organisation de leur bien-être était suffisamment bonne, les troupes seraient moins disposées à visiter les cafés et restaurants civils ", le Comité espérait contenir et contrôler le comportement public des troupes $^{33}$. Des méthodes récréatives similaires furent adoptées pour les troupes américaines, notamment par le United Services' Club ${ }^{34}$. Néanmoins, les inquiétudes de l'opinion publique iranienne persistèrent, incitant la police urbaine de Téhéran à prendre de nouvelles mesures. Une lettre confidentielle de la légation des ÉtatsUnis adressée au Département d'État révèle qu'en juin 1943, une réunion eut lieu "au quartier général militaire de la ville de Téhéran »; les propriétaires de plusieurs restaurants et hôtels y furent conviés, dans l'optique de leur demander la suspension de toute activité de danse européenne ayant lieu dans leur établissement. Le haut dignitaire religieux à l'initiative de cette décision, présent lors de la réunion, clarifia les motivations de sa requête lorsque les restaurateurs exprimèrent leur inquiétude face à leur perte de revenu probable: "Nous ne voulons pas que nos femmes iraniennes dansent avec des étrangers ", lança-t-il ${ }^{35}$. Néanmoins, comme le montre un rapide examen de la presse locale, les cours de danses de société et les établissements avec une forte présence américaine restèrent très populaires, bien qu'en parallèle ils continuent à être désignés comme "des espaces de criminalité et de malfaisances », suscitant ainsi encore plus d'inquiétude à propos du contact sexuel entre soldats et

33 Première réunion du Comité social (Welfare Committee) de la sous-zone de Téhéran, 10 décembre 1942, Archives britanniques, WO-169-7696.

34 Les photos des activités du United Services' Club au Camp Amirabad à Téhéran nous fournit des informations précieuses sur la programmation récréative de l'armée américaine en Iran. Cf. Records of the Office of War Information, 19261951. Photographs of the Allies and Axis, 1942-1945. AEF-Iran-Entertaining. Archives nationales de College Park, MD.

35 Bertel Kuniholm, légation des États-Unis, au Département d'État, 7 juin 1943 ; No. 840, volume confidentiel. Récupéré aux Archives nationales de College Park, MD. 
Iranien.ne.s ${ }^{36}$. Un article fictif paru dans l'hebdomadaire Taraqqi et intitulé "Chasser rue Lalehzar: comment les femmes chics se font dérobées », dépeignait le viol d'une jeune mariée par des soldats américains comme la conséquence de sa décision naïve de monter dans une Buick $41^{37}$. L'histoire se terminait avec une observation plus générale: «Chaque jour, des douzaines de femmes iraniennes perdent leur chasteté de cette façon ».

Les lieux où les soldats alliés et les Iraniennes dansaient en couple ne se limitaient pas aux espaces de divertissement. Dans ses mémoires, La Danse de la rose et du rossignol (2002), Nesta Ramazani signale avoir assisté à une soirée de danse au camp militaire américain d'Amirabad : les femmes qui souhaitaient danser avec les soldats devaient attendre à des stations de bus spécifiques, afin de s'y faire conduire. Selon son récit, les résidents lancèrent des injures aux femmes se trouvant à ces arrêts de bus, usant des invectives "putains », «donneuses de chatte » et «traittres », indépendamment d'ailleurs de la nationalité ou la religion de ces femmes ${ }^{38}$. Rajoutant à l'outrage, un article du magazine laïc Taraqqi enfonça le clou quand il décrit ces femmes comme étant «meilleur marché [que] les "prostituées de Monte Carlo" »" Les revues d'orientation religieuse partageaient ces jugements, bien qu'elles retraçaient toujours le problème jusqu'à l'interdiction du port du voile et aux pratiques attribuées à une modernité d'imitation, avec toutes les conséquences néfastes que cela comprenait. Un article datant de 1944 et intitulé «Les femmes et le hijab », se lamentait ainsi :

[...] nous avons atteint un moment où les femmes iraniennes considèrent maintenant le fait de couvrir leur corps et de suivre les règles de la chasteté comme des coutumes arriérées, et regardent au contraire les tenues dénudées, les activités de danse et le fait d'embrasser des

36 H. Karbasi, «Salun-i raqs, kilass-i dars » [Salon de danse, salle de classe], Parchami Islam, 2 Urdibihisht 1324/22 avril 1945, p. 2, p. 4 [citation en 2].

37 «Shikar dar khiaban-i Lalehzar, khanum-ha-yi shik ra chigunah mirubayand» [Chasser rue Lalehzar, ou comment les femmes chics se font dérobées], Taraqqi 8, 21 Aban 1324/11 novembre 1945, p. 18.

38 Ramazani 2002 : 160-161.

39 «Sarbazan- $i$ amrika'i az iran chih mibarand?" [Quel souvenirs les soldats américains rapporteront-ils d'Iran ?], Taraqqi 156, 17 Day 1324/8 janvier 1945, p. 16. 
étrangers comme représentant la liberté et la civilisation; elles ressentent de la fierté à être des instruments de loisir et de plaisir pour ceci et cela ${ }^{40}$.

\section{Le Téhéran qui danse « brûle " : de la métaphore à la réalité}

Le départ des troupes britanniques et américaines en octobre 1945 ne mit fin ni aux inquiétudes sur les danses de couples ni au courants religieux s'opposant au dévoilement des femmes, les deux pourtant reliés à une influence étrangère. Un auteur religieux avançait par exemple que les Européens, encouragés par les hommes politiques qui avaient dévoilé les Iraniennes de force, avaient détruit l'honneur (namus) des hommes iraniens ${ }^{41}$. Un autre écrivait :

Protéger le namus est l'une des premières responsabilités de tout individu iranien... Le namus est important pour la société car la survivance des générations (futures) en dépend ${ }^{42}$.

Dans ce contexte, les Iraniennes qui dansaient avec un homme ajnabi - qu'il soit iranien ou étranger - entraient en conflit direct avec l'idée de protection de l'honneur, particulièrement lorsque circulait la notion qu'une femme n'était pas autorisée à prendre part à la danse (le terme français désignait les danses de couple), même en présence de son mari :

Il n'y a aucune raison pour que des hommes et des femmes étrangers l'un à l'autre dansent ensemble dans ce qu'ils nomment la danse, mis à part la corruption des mœurs (fisad-i akblaq)... Il est absurde qu'en danse, mari et femme doivent ne pas s'associer ${ }^{43}$.

Un homme qui emmenait sa femme au bal et qui la regardait pendant qu'elle dansait avec d'autres hommes était décrit tour à tour comme biqayrat (manquant de zèle), comme un gigolo, et comme un proxénète

40 «Zanan va hijab » [Femmes et hijab], A'in-i Islam 16, 9 Tir 1323/30 juin 1944, p. 1-3.

41 Yadullah Niyazmand-Shirazi, «Zan dar ijtima’i diruz va imruz, 4 » [Les femmes d'hier et d'aujourd'hui dans la société, 4], Nur-i Danish, 12 Azar 1326/4 décembre 1947, p. 289-290.

42 «Tazahurat-i ijtima’i », Dunya-yi Islam, 10 Mihr 1327/2 octobre 1948, p. 3.

43 Muhamd-Ali Ansari, «Parah sazid ayah ha-yi hijab» [Déboulonner les conséquences du voile], Dunya-yi Islam, 28 Azar 1326 /20 décembre 1947, p. 1, p. 2 [citation en 2]; "Fisad-i akhlaq" [Corruption morale], Parcham-i Islam, 3 Azar $1328 / 24$ novembre 1949 , p. 1, p. 4 [citation en 4]. 
(les termes qurumsaq et dayyus étaient tous deux employés) ${ }^{44}$. Les danseurs de sexe masculin étaient considérés comme moralement dépravés et désireux d'exploiter la naïveté des femmes iraniennes :

En dansant le pasodoble, le foxtrot, le tango, la rumba et la valse, ils cherchent à satisfaire leurs désirs sexuels - ceci est en effet une nouvelle technique de masturbation ${ }^{45}$.

Suivant ce raisonnement, le quartier Lalehzar qui accueillait la majorité des établissements de danse, fut représenté comme un quartier criminel où des playboys ivres et peu honorables déambulaient alors que des femmes à demi nues adoptaient un comportement si impudique qu'il était difficile de les différencier de prostituées ${ }^{46}$. Les journalistes qualifièrent Téhéran de «centre pour coureurs de jupons » (kanun-i zanparastan) où des images d'actrices et de danseuses s'affichaient à chaque coin de rue ${ }^{47}$. La ville fut jugée comme un "cimetière d'impuretés » (qabristan-i pakdamami), une " école d'imitation » (madrasab'i taqlid), et « un marché d'impudeurs » (jaygah-i furush-i hujb va haya) où l'on s'adonnait à la raqs (un style de danse locale), la danse et l'alcool ${ }^{48}$. Dans un article de 1947 de la revue Parcham-i Islam, l'auteur maintenait que la danse ainsi que d'autres formes de «corruptions » avaient envahi la vie publique de Téhéran. Il notait :

Nous regardons Téhéran danser dans le feu de la corruption, du plaisir et des crimes - mais ce n'est pas seulement Téhéran mais l'Iran et la totalité du monde musulman qui danse dans ce feu... Téhéran danse et brûle... ${ }^{49}$

44 Ahmad Shari'ati, "Qurumsaq-ha ra bishinasid» [Faites la connaissance des proxénètes !], Dunya-yi Islam 48, 2 Aban 1326/20 octobre 1947, p. 3 ; «Dushizigan... » [Jeunes femmes...], Dunya-yi Islam 28, Urdibihisht 1326/mai 1947, p. 4.

45 Munsif Yaraqchi, «Sukhani dar piramun-i hijab » [Quelques mots sur le hijab], Dunya-yi Islam, 12 Farvardin 1326/2 avril 1947, p. 2.

46 «Shumal va junub» [Nord et sud], Parcham-i Islam, 2 Khurdad 1325/23 May 1946, p. 1 ; Yadullah Niyazmand-Shirazi, «Zan dar ijtima’i diruz va imruz », p. 52.

47 Sayyad Jalal Kashani, «Shahr-i khuda-parastan ast ya kanun-i zanparastan » [Estce la ville des adorateurs de Dieu ou des adorateurs de femmes ?], Dunya-yi Islam, 1 Khurdad 1327/22 mai 1948, p. 4 ; Shir-khuda'i, "Shahr-i fajayi », Parcham-i Islam, 26 Day 1325/17 décembre 1946, p. 3.

48 H. Karbasi, «Tihran dar atash-i fisad misuzad » [Téhéran brûle dans le feu de la corruption], Parcham-i Islam, 12 Azar 1326/4 décembre 1947, p. 1-2.

49 Ibid. 
Des attaques moins virulentes contre les pratiques de danse de société se retrouvaient dans les travaux d'écrivains d'idéologie laïque. Surur Afkhami, une auteure vraisemblablement féministe, avisait les jeunes femmes iraniennes de dépenser leur énergie à se battre pour leurs droits, plutôt que de perdre leur temps avec la mode, le maquillage et la maitrise de pas de danse. Elle conseillait ses lectrices de se focaliser sur l'essentiel :

Si vous et moi nous entrainions au swing pour encore cent ans, même si nous arrivions à un point où nous devenions meilleures que les Parisiennes [?] en samba, nous resterions les mêmes opprimées (tu-sarikhur) que nous sommes aujourd'hui ${ }^{50}$.

Les revues publiées entre 1950 et 1979 révèlent qu'une réelle frénésie pour les danses de société perdura jusqu'à la Révolution, même si les tendances américaines remplacèrent peu à peu l'engouement pour les tendances françaises. La publicité du film Cha Cha Cha, projeté en 1957 au cinéma de Téhéran (fig. 6 - cf. cahier couleur), illustre la popularité continue de ces danses. De même, un sondage réalisé en 1962 confirme la prévalence du tango, du Madison, du twist et du rock n'roll en Iran - bien qu'un certain nombre de femmes avouèrent ne pas apprécier le tango dû au contact très proche que celui-ci impliquait avec des partenaires aléatoires ${ }^{51}$. Par moments, l'état tenta de contrôler les activités de la jeunesse iranienne, par exemple en interdisant le rock n'roll dans les boîtes de nuit de Téhéran dans les années 1950, ou en interdisant l'accès d'élèves du secondaire à des clubs de danse durant la journée ${ }^{52}$. De telles mesures s'expliquaient généralement par l'infiltration de trafiquants de drogue dans les clubs de danse ${ }^{53}$.

50 Surur Afkhami, «Man bara-yi suing mimiram, shuma chitor? » Je meurs pour le swing, pas vous ?], Taraqqi 10/8, 28 Shahrivar 1329/19 septembre 1950, p. 11, p. 21.

51 «Iftitah-i salun-i zimistani-i sinama tihran » [L'ouverture de l'établissement d'hiver du Cinéma de Téhéran], Majallab Sinama, 1/2, 24 Mihr 1336/16 octobre 1957, p. 16 ; «Raqs dar iran » [La danse en Iran], Majallab Sinama 32, 1341/1962, p. 21.

52 Voir "Sanad-i shumarah'i 153 » [Document no. 153] dans Ali-Akbari Baigi et I. Muhammadi (ed.) Asnadi az Musiqi, Sinama va Ti'atr dar Iran, 1300-1357 [Documents sur la musique, le cinéma, et le théâtre en Iran, 1300-57], vol. 1-3, Téhéran, Sazman-i Chap va Intisharat-i Vizarat-i Farhang va Irshad-i Islami, 1379/2000, vol. $2: 730$.

53 «Rukhsar az shikufah’i naw taqaza-yi paygiri va mujazat hosseinpur az zindan-i 
Avec l'expansion continue des sites de loisirs et des pratiques et moyens de divertissement, ainsi qu'avec la dominance américaine de la scène politique iranienne, la controverse sur la danse changea de ton et d'intensité. Cela était essentiellement dû à l'intensification de l'influence américaine à la suite du coup d'État de 1953 soutenu par le MI6-CIA. Ayant d'abord éclaté après la Première Guerre mondiale, cette polémique fut peu à peu marginalisée puis assimilée par le discours anti-impérialiste et révolutionnaire plus large qui ciblait les États-Unis davantage que l'Europe. Le sexe, la danse, l'alcool et les drogues étaient dorénavant vus comme des armes impérialistes avec lesquelles les États-Unis tentaient d'empêcher les pays «déshérités » (mustaz'af) et affaiblis de se développer ${ }^{54}$ (fig. 7).

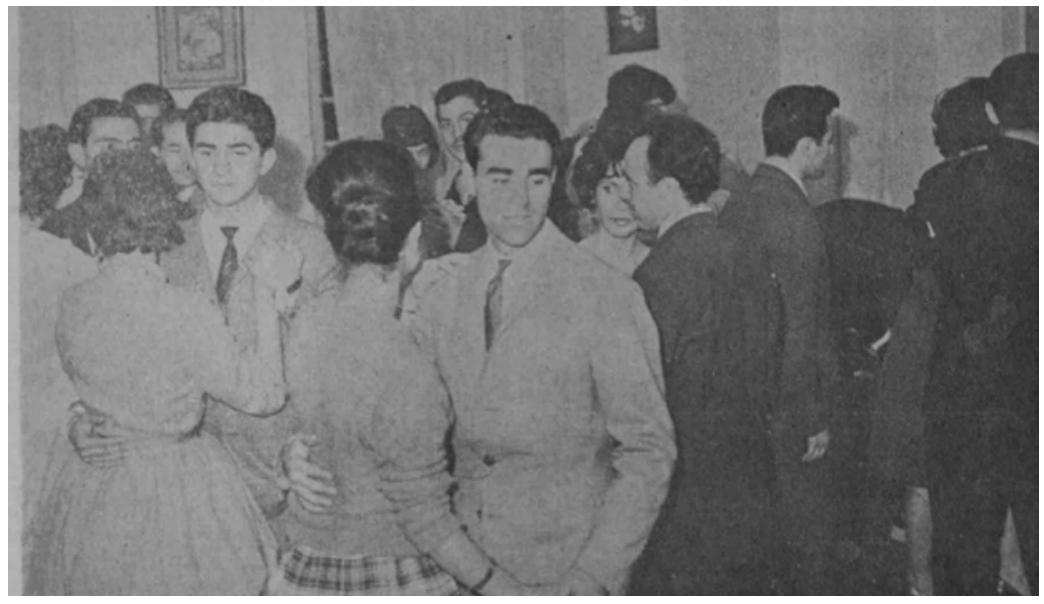

Fig. 7. Soirée dansante à Téhéran en 1960 (d'après Farrukh Safavi, «Raqs dar jam'iah'i ma» [La danse dans notre société], Iran-i Abad, Azar 1339 / novembre 1960, p. 69-72, ill. page 72).

shumarah'i yik qasr» [Rukhsar de Shikufah'i Naw demande la punition de Hosseipur retenu à la prison de Qasr], 4/2417/405/1/3, fonds Muzah va Markaz-i Asnad-i Majlis-i Shawra-yi Islami [Bibliothèque, musée, et centre documentaire de Majlis]

54 Ahmad Bihishti, «Mustaz'af kist? " [Qui sont les déshérités ?], Maktab-i Islam 20/2, Urdibisht 1359/avril-mai 1980, p. $22-28$ [citation en 25]. 
Néanmoins, l'engouement pour les danses de société dans les années 1940 fut réel et laissa des traces qu'il est important d'examiner. L'image controversée des femmes iraniennes enlacées dans les bras de l'occupant étranger tout comme le discours anti-obscénité profondément genré auquel cette polémique donna lieu - attaquant la virilité des hommes iraniens «modernes » en fusionnant nationalisme et chasteté féminine - ne furent pas sans conséquence. Le discours anti-obscénité devint en effet partie intégrante de la Révolution de 1979. Le «feu» révolutionnaire cibla délibérément les lieux "d'obscénité », parmi lesquels on comptait un certain nombre de cabarets incendiés au cours de la Révolution (fig. 8). En ce sens, la métaphore de Téhéran qui « danse et brûle » citée dans le texte de 1947 plus haut, devint une réalité tangible au lendemain de l'installation du nouvel ordre socio-politique.

\section{Traduit de l'anglais par Aurélie PERRIER}

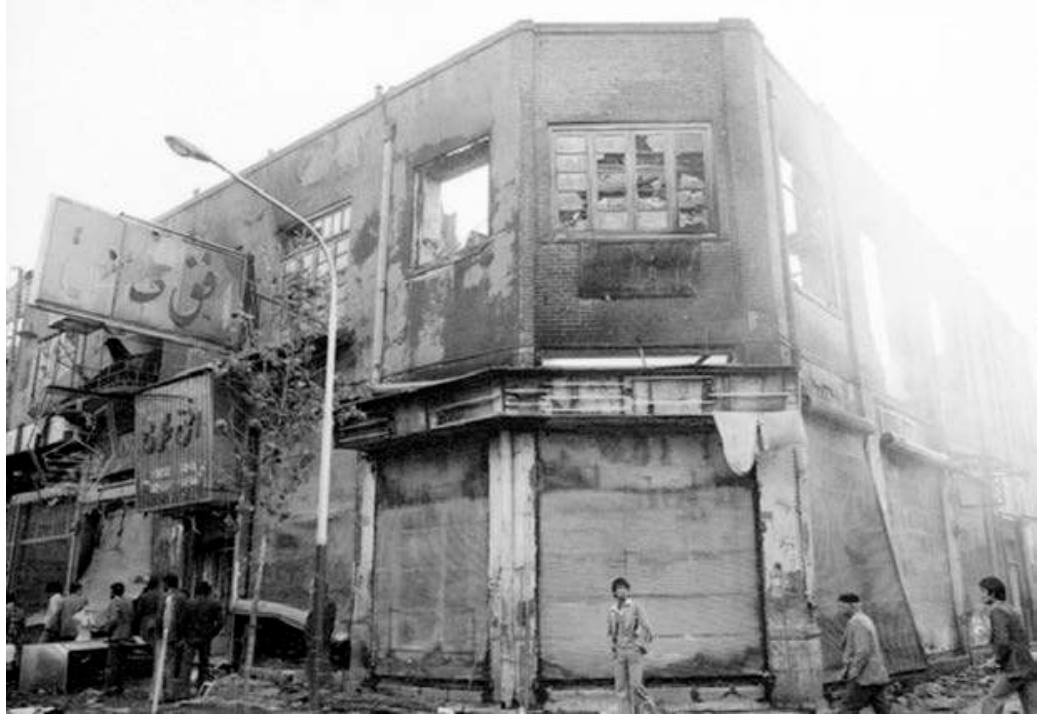

Fig. 8. Photographie du Cabaret Ufuq Tala’i dans le quartier Lalehzar, après qu'il ait été brûlé (Lalehzar Digital Archives). 


\section{Bibliographie}

CLAIRE Elizabeth, 2013, «Inscrire le corps révolutionnaire dans la pathologie morale: la valse, le vertige, et l'imagination des femmes », Orages. Littérature et culture 1760-1830, 12 : «Sexes en Révolution » (sous la dir. de Florence LOTTERIE \& Pierre FrANTZ), p. 87-109.

—, 2017, "A moral defence of the Regency Ballroom - vide Wilsons Rooms », European Drama and Performance Studies, 8: «Danse et morale. Une approche généologique », Marie GLON \& Juan Ignacio VALlEJOS (dir.), p. 163-192.

Guran Hiva, 1360/1981, Kushish-ha-yi Nafarjam: Sayri dar Sad Sal Ti'atr-i Iran [Futile endeavours: a journey across one hundred years of theater in Iran], Téhéran, Intishahrat-i Agah.

Јасотот Sophie, 2013, Danser à Paris dans l'entre-deux-guerres, Paris, Éd. Nouveau monde.

Kazmizadeh Iranschahr Hussein (ed.), 1363/1984, Majalleh Iranchahr, Téhéran, Eqbal.

Kouhestani-Nejad Mas'ud (ed.), 1381/2002, Guzৃidab'i Asnad-i Namayish dar Iran, az Inqilab-i Mashrutiyat ta 1304 [Sélection de notices sur le drame iranien, de la Révolution constitutionnelle jusqu'à 1925], vol. 2, Téhéran, Intisharat-i Sazman-i Asnad-i Milli-i Iran.

—, (ed.), 1384/2005, Musiqi dar 'Asr-i Mashrutah [La musique pendant l'ère constitutionnelle], Téhéran, Mehrnamag.

Meftahi Ida, 2016, Gender and Dance in Modern Iran: biopolitics on stage, London, Routledge.

NAFICY Hamid, 2011, "International haggling over Iranian public screens », $A$ Social History of Iranian Cinema, vol. 2: The industrializing years, 1941-1978, Durham, Duke University Press, p. 1-48.

Ramazani Nesta, 2002, The Dance of the Rose and the Nightingale, Syracuse, Syracuse University Press. 